\title{
On the EU Approach for DEMO Architecture Exploration and Dealing with Uncertainties
}

\author{
M. Coleman ${ }^{\mathrm{a}, \mathrm{b}, *}$, F. Maviglia ${ }^{\mathrm{a}}$, C. Bachmann ${ }^{\mathrm{a}}$, J. Anthony $^{\mathrm{b}}$, G. Federici $^{\mathrm{a}}$, M. Shannon ${ }^{\mathrm{a}, \mathrm{b}}$, R. Wenninger $^{\mathrm{a}, \mathrm{c}}$ \\ ${ }^{a}$ EUROfusion Consortium, Boltzmannstraße 2, 85748 Garching, Germany \\ ${ }^{b}$ CCFE Fusion Association, Culham Science Centre, Abingdon, Oxfordshire OX14 3DB, United Kingdom \\ ${ }^{c}$ Max-Planck-Institut für Plasmaphysik, 85748 Garching, Germany
}

\begin{abstract}
One of the difficulties inherent in designing a future fusion reactor is dealing with uncertainty. As the major step between ITER and the commercial exploitation of nuclear fusion energy, DEMO will have to address many challenges - the natures of which are still not fully known. Unlike fission reactors, fusion reactors suffer from the intrinsic complexity of the tokamak (numerous interdependent system parameters) and from the dependence of plasma physics on scale - prohibiting design exploration founded on incremental progression and small-scale experimentation. For DEMO, this means that significant technical uncertainties will exist for some time to come, and a systems engineering design exploration approach must be developed to explore the reactor architecture when faced with these uncertainties. Important uncertainties in the context of fusion reactor design are discussed and a strategy for dealing with these is presented, treating the uncertainty in the first wall loads as an example.
\end{abstract}

Keywords: DEMO, systems engineering, architecture, uncertainty, design exploration, first wall

\section{Introduction}

At this pre-conceptual stage of the EU DEMO design process, there are many technical uncertainties in the design activities with which we are obliged to contend. This paper presents an outline strategy for tackling uncertainties in the DEMO design, and an investigation into a particularly challenging design uncertainty: that of the first wall (FW) loads.

It is important to continuously re-evaluate the direction of the design in the pre-concept phase by assessing and comparing alternative configurations against a range of criteria. As more information relating to DEMO design points is generated, the baseline design will evolve, and may need to take some stepwise developments in order to reach an attractive point in the design space. Gradual progressions are relatively well dealt with by traditional systems engineering approaches and design space exploration techniques; however in DEMO some key aspects do not lend themselves so readily to parameterisation and response surface type optimisations.

\section{The nature of uncertainties}

The EU DEMO design is inherently fraught with uncertainties. The majority of these (and those that are the most problematic) stem from insufficient knowledge in both physics and engineering aspects of the tokamak; these are termed epistemic uncertainties. Naturally, there are "known unknowns" and "unknown unknowns"; uncertainties that we are aware of and those which we are not yet aware of - both of which have to be dealt

${ }^{*}$ Corresponding author. Tel.: +49 (0)89 32994209 matti.coleman@euro-fusion.org with, where possible. On the other hand, aleatory uncertainties arise from random effects and, although they can remain unquantified, they cannot be eliminated. A real-world uncertainty is typically a mixture of these two flavours of uncertainty: e.g. the material properties of a novel alloy are not known until they are characterised - even after which some statistical variation will inevitably remain.

In principle, epistemic uncertainties are reducible: through either theory or experiment, one could technically devise a means to eliminate a known lack of knowledge, and one may also expect to discover further shortfalls in knowledge, of which we were previously unaware. In fusion research and development (R\&D) however, the timely elimination of epistemic uncertainties is in many cases infeasible. The dependency of tokamak plasma physics on scale and the nature of the various phenomena that require further study (e.g. fusion neutron effects on materials) are such that replicating the DEMO operating environment is an expensive and complex endeavour. Indeed, the construction and operation of ITER is strategically oriented to reduce many of the epistemic uncertainties in plasma physics, technology, tokamak design and operation, and tritium breeding. In the meantime, the focus must be on dealing with the existing uncertainties, whilst simultaneously progressively reducing them: work which is typically done through strategic $\mathrm{R} \& \mathrm{D}$ programs throughout the DEMO work packages.

\section{Systems engineering approach for dealing with uncer- tainty}

Traditional systems engineering practices have tended to be developed for individual systems with relatively closed boundaries: clear and detailed requirements, few - if any - complex 
interfaces, few external factors of influence, etc. DEMO, however, is altogether a very different type of system; there are few known and detailed requirements, numerous interfaces and interdependencies, and is in general characterised by high complexity and uncertainty. A much-loved metaphor for these two types of systems (and those who solve them) is that of the watchmaker and the gardener. The watchmaker knows exactly what the requirements of the system are, is already aware of a viable solution, and knows how to go about delivering it. The gardener however, must operate in an uncertain environment which is liable to change, and where each aspect of the system affects the other, sometimes unpredictably. As such, the gardener cannot operate with a fixed solution in mind and must instead work towards maximising the likelihood of achieving a successful outcome.

It is thus that we must proceed with DEMO, and for such an endeavour to be successful, it is vital that a systematic approach is developed and implemented, in particular regarding the numerous uncertainties at this relatively early stage of development.

\subsection{Strategic technical approach and baselining}

For DEMO, progressive steps must be taken in paper-based reactor studies, supported by $\mathrm{R} \& \mathrm{D}$. In the EU, these are coordinated by EUROfusion issuing physics and engineering baselines for study. These baselines are generated from the PROCESS system code output and later in CAD, and are based on meeting the DEMO plant requirements $[1,2]$. These elements form part of the DEMO Plant Architecture Model (PAM), which is a definition of all of the entities of the plant and the relationships between them: geometry, functions, requirements, etc. It plays a pivotal role in the behaviour and performance of the plant; careful definition and manipulation of the architecture are paramount to the success of the plant as a whole.

The baseline PAM constitutes a snapshot of the proposed reactor design, which is periodically updated to incorporate new information. The complexity of DEMO is such that, in many cases, changes in a single parameter in one system can cause significant fluctuations in the boundary conditions of another; the DEMO sub-systems cannot be meaningfully developed in isolation. Due to the numerous uncertainties and the strong interdependencies between the constituent systems, it is necessary to fix a single architecture for study to enable coherent DEMO sub-system development. This temporarily defines many boundary conditions and allows the investigation of this design point, populating the multi-parameter design space with some concrete information, which remains useful even after the architecture is subsequently changed.

Currently, the EU-DEMO 2015 baseline PAM consists of a single-null (SN) tokamak based on a vertical maintenance scheme, with 18 toroidal field coils and tokamak sectors [1]. However, in order to mitigate technical risks that become apparent in the light of new information, alternatives to this baseline must be studied in parallel - without perturbing baseline design point studies.

\subsection{A framework for treating uncertainties from an architec- tural perspective}

Whilst there are uncertainties within each sub-system, some of these transcend the sub-system boundaries and a problem arises when the presently posited DEMO architecture is not viable over a wide range of a specific technical uncertainty. This is particularly challenging when across the range of a physics uncertainty, step changes in technology or strategy are required, i.e. the response of a system to an input parameter is a discontinuous function. For example, beyond a peak field at the conductor of 10-11 T, NbTi is no longer an appropriate material choice for fusion magnet superconductors and a different technology (e.g. $\mathrm{Nb}_{3} \mathrm{Sn}$ ) is required.

In the case of important physics uncertainties which have a significant effect on the DEMO design, it is important to investigate the implications from a system perspective, see also [1]. This is in part to understand the importance of the uncertainty, but also to determine whether within this range of uncertainty, there is a step change in response or a feasibility limit. Such information can be captured through the creation and control of requirements which may be imposed on the systems dealing with the uncertainty, but may also be imposed upon the uncertainty itself, e.g. at some limit a load is no longer acceptable without engendering engineering solutions that are either impossible or have unacceptable negative consequences for the DEMO plant.

In parallel to reducing these intrinsic uncertainties, it is important to consider a reasonable range of the uncertainty in a parameter (based on existing estimates and comparable systems), to examine the architectural implications. As an uncertainty threatens the feasibility of the baseline PAM, alternative PAMs are developed and compared against the baseline for a selection of evaluation aspects (EAs) pertinent to the uncertainty being considered (e.g. TBR, plant efficiency, technology readiness, etc.) in a representative Pugh-like matrix (Figure 1). To enable decisions to be taken, EAs must be agreed and weighted against each other, preferably based on systems engineering principles through modelling of the system space. Where possible, mathematical relationships between the relative importance of parameters and the comparison of architectures should be derived, however expert opinion will form an integral part of any comparison or decision made through this framework.

The systems which are identified as being the most heavily affected by an uncertainty or modification are studied first, to prevent too many resources being invested in studying configurations which are clearly unfavourable. The modification of the DEMO architecture is usually done with incomplete information; in the pre-conceptual design phase it would be impossible (with finite resources) to fully quantify the response of any of the DEMO sub-systems at a given design point, let alone all of them for multiple different architectures. The conundrum is again that of the gardener; the architecture must be modified such that the plant is given the best possible chance to meet the high-level requirements based on the presently available information.

As an epistemic uncertainty is reduced and the field of doubt is narrowed, confidence in a refined range of the uncertainty is 


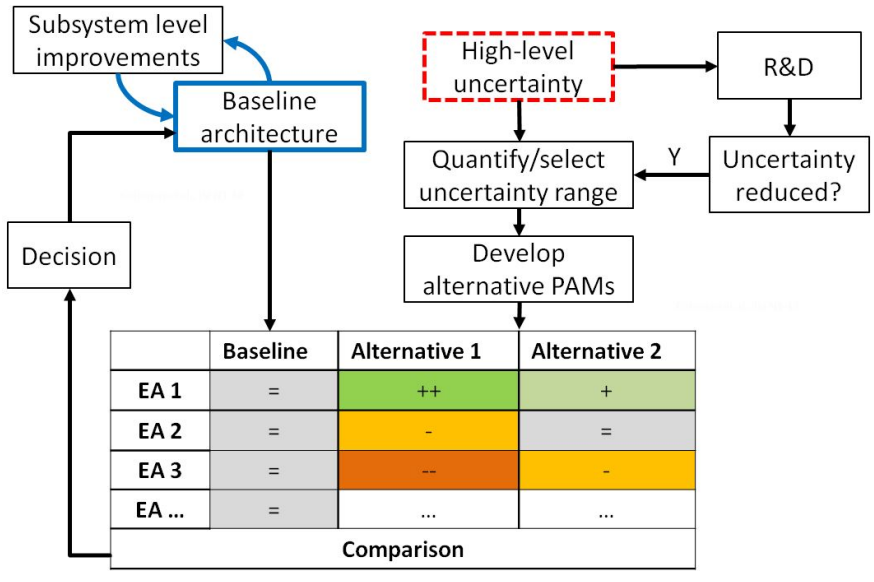

Figure 1: Addressing high-level uncertainties through consideration of alternative DEMO architectures; these are compared against the baseline, leading to a decision on whether or not to modify it. In parallel the uncertainty itself is also studied, in the hope it is reduced, potentially influencing the PAMs being developed and the decision itself.

increased and the baseline may be progressively modified. Ideally, this would occur to the point where alternative architectures no longer need to be considered; parameterised modelbased approaches become more readily applicable, e.g. for a parameter $P$ with a reduced uncertainty range $u, E A 1=$ $f(P \pm u / 2), E A 2=g(P \pm u / 2)$, etc.

\section{Modifying the EU DEMO architecture in response to un- certainties}

\subsection{Tritium self-sufficiency}

Faced with the uncertainty of the DEMO baseline to meet the high-level requirement of tritium self-sufficiency, steps have recently been taken to modify the distribution of the in-vessel components to achieve a higher tritium breeding ratio (TBR). This was incorporated in the latest DEMO baseline model, with preliminary results indicating an increase in potential TBR of approximately 6\% [3]. While questions remain regarding the divertor system and its ability to meet all of its requirements within the shrunken space envelope (in particular the likely increased loads on the blanket FW where the divertor baffles were previously), at the time of the decision no strong driver for retaining the size of the ITER-like divertor was identified.

\subsection{Aspect ratio}

The parameter space for DEMO is still being explored. The aspect ratio was identified as one of the most important parameters which was still relatively unconstrained. In 2014, studies were carried out in various areas to understand the effects of aspect ratios between 2.6 - 3.6 on the pulsed DEMO design. For a given major radius, a lower aspect ratio implies a larger plasma volume and lower toroidal field, resulting in a higher TBR, better vertical stability [4], and lower disruption forces, amongst other benefits. Although some aspects relating to a decision on the aspect ratio were not assessed in depth (e.g. cost, maintainability), the DEMO aspect ratio was changed from 4 to 3.1 in recognition of a general trend despite preliminary results indicating lower aspect ratios are less favourable from a maintenance perspective [1]. The importance of investigating multiple design points in the pre-conceptual design phase cannot be overstated; more information relating to the choice of DEMO aspect ratio is being collated and may result in further modifications.

\subsection{Blanket maintenance}

The uncertainty in the capability of remote maintenance systems to reliably and safely manipulate the blanket segments weighing tens of tons in one of the harshest conceivable environments for robotic systems has serious ramifications for the layout of in-vessel components and the entire DEMO plant. Accurate control and positioning of the massive blanket segments in a radiation environment which essentially prohibits the use of sophisticated viewing and sensing equipment is a considerable technological step from present capabilities [5]. Were some limit load or installation/clearance tolerance identified, this could in future lead to a radically different blanket segmentation, affecting the plant performance and architecture.

\section{FW heat loads and architectural/system implications}

Perhaps the most critical epistemic uncertainty in DEMO due to its significant impact on the in-vessel components and the architecture of the plant as a whole - is the uncertainty in the FW loads. This particular aspect is discussed here in more detail as an example of the application of the approach for dealing with uncertainty described above.

The plasma-facing FW will be exposed to heat and particle fluxes resulting from various phenomena originating in the plasma. The steady-state FW loads are comprised of contributions from radiation from the plasma core, neutrals, fast particles, and radiation and thermal charged particles from the scrape-off layer (SOL) which includes far-SOL transport phenomena (blobby filaments).

The difficulty lies in our currently limited understanding and predictive capability of these loads. In a DEMO machine, core radiation and neutron flux can be calculated with a good degree of confidence (see e.g. [4]), however SOL phenomena are far harder to predict; uncertainties in the power decay length, $\lambda_{\mathrm{q}}$ - estimated to lie in the range of $40-170 \mathrm{~mm}$ for the far SOL [6], and the distribution of SOL power between radiation and thermal particles, and between near and far SOL transport phenomena leads to a large cumulated uncertainty. The shape of the FW (in 2D and 3D) has a significant impact in particular for the thermal charged particle loads, and must be designed with the operational plasma equilibria in mind. An improved process for shaping the FW is being developed and has to some extent been adopted [8]. It is clear however that there will be a region at the top of the machine where the FW loads will be higher where some magnetic flux surfaces are inevitably intersected by the FW, which cannot be fully conformal for all equilibria, 
leading to peaked thermal charged particle loads. Manufacturing restrictions on large, modular FW panels also imply singlecurvature panels in most places, meaning perfect FW conformity with an equilibrium is in any case challenging.

Preliminary estimates for the core radiation $\left(\sim 0.3 \mathrm{MW} / \mathrm{m}^{2}\right.$ [4]), and scans for SOL radiation and thermal particle loads for a range of power distributions and $\lambda_{\mathrm{q}}$ [9] indicate loads at the top of the machine will potentially exceed $1.5 \mathrm{MW} / \mathrm{m}^{2}$ for the first iteration of 3D FW shapes.

The predictive capability for transient loads due to edgelocalised modes, confinement transients, disruptions, and other events in DEMO are limited and this remains an important uncertainty. Transients, alongside various other considerations, such as manufacturing and assembly tolerances, magnetic field asymmetries, and the control of the plasma will drive up the design heat load of the FW. Work is under way to develop a DEMO wall load specification which will provide poloidally resolved estimates of a wide range of static and transient loads [4].

At present, in light of the many uncertainties mentioned above, it is perhaps not inconceivable to take an upper limit for the design heat load at the top of the machine of the order of 5-7 MW/ $/ \mathrm{m}^{2}$. As a reference, the ITER allowable heat load for this part of the machine is $4.7 \mathrm{MW} / \mathrm{m}^{2}$, which is the result of considering many physics load cases and penalty factors for engineering uncertainties [7].

\subsection{SN baseline}

In the DEMO baseline, the FW is currently assumed to be mechanically and hydraulically integrated with the blanket, and as such is required to have a service life greater or equal to that of the blanket (replacement of the FW alone is not presently considered)[3]. In addition to withstanding the heat loads originating in the plasma, the FW must also enable efficient power extraction (approximately $30 \%$ of the power deposited in the blanket is extracted via the FW), and must not overly compromise the tritium breeding performance of the blanket. These requirements impose restrictions on the operating temperature, pressure drop and mass flow rate, thickness, and material and coolant composition of the FW. Studies have shown that for integrated Eurofer-based FW technologies, design heat loads of $\sim 1 \mathrm{MW} / \mathrm{m}^{2}$ for He-cooling and $\sim 1.5 \mathrm{MW} / \mathrm{m}^{2}$ for $\mathrm{H}_{2} \mathrm{O}$-cooling are achievable [8].

Means of optimising the plasma equilibria and the FW shape in the baseline to reduce the loads on the FW (without changing architecture) are also being pursued. A sensitivity study was performed on the impact of the geometric position of the upper magnetic null on the localisation and peak of the heat flux density [9]. The secondary inactive null was moved closer and further away to the plasma with respect to the original position, keeping the plasma shape as close to the original as possible. The upper heat loads were found to be lower for equilibria with secondary nulls further from the chamber, with the power spread across a larger area, while the heat loads increase with the secondary null closer to the chamber, with the power focusing on a smaller area (Figure 2). While the latter case is worse in terms of localised peak heat flux, it may be convenient to

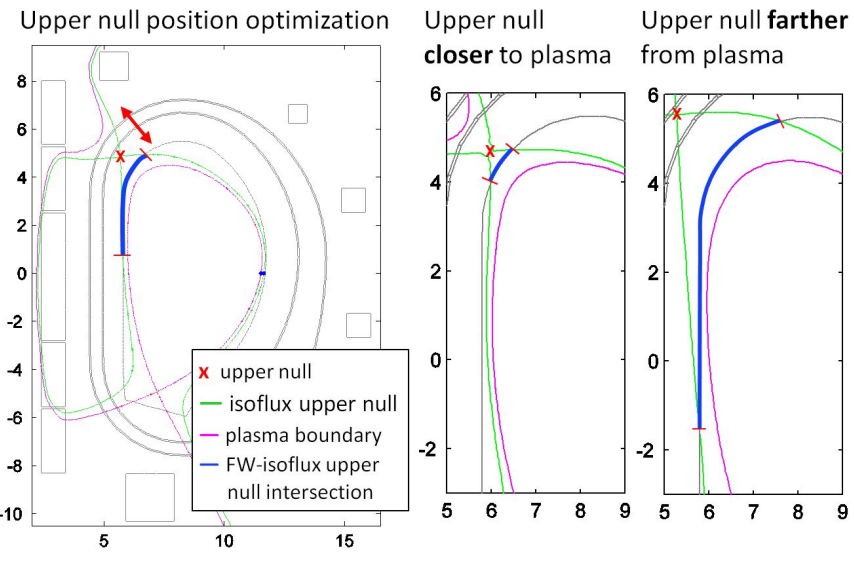

Figure 2: Sensitivity scan of the upper x-point position. The intersection of upper-null iso-flux curve with first wall is larger with the magnetic null further from the vessel, but with smaller peak heat loads.

limit the area where dedicated high heat flux components need to be installed.

Regardless of the exact value of the required FW design heat load at the top of the machine, it is clear that the present architecture and technologies are only viable over a small portion of the posited range of uncertainty, particularly in the case of a He-cooled blanket.

As such, alternative tokamak configurations must be investigated at this stage, to study mitigation options and steer the architecture of the DEMO plant in a direction considered most likely to ensure a viable plant design. Two generic configurations will be considered and compared against the SN baseline: a SN tokamak with local HHF panels integrated at the top of the machine, and a double-null (DN) configuration. Schematic representations of these three architectures are shown in Figure 3. Other significant architectural modifications are also considered outside of this exercise, in particular alternative magnetic configurations and plasma exhaust strategies.

\subsection{SN configuration with dedicated HHF FW panels}

Integrating a set of toroidally continuous high heat flux (HHF) components in the vertical port to deal with the higher heat fluxes experienced by the FW in this region of the machine could prove to be a viable mitigation strategy. Dedicated HHF components could enable effective machine protection, but since they will be thicker than the nominal FW, cooled at low temperatures, and may have a lower lifetime, they are likely to degrade the TBR, the plant efficiency, and the availability, respectively. Several permutations of this alternative are conceivable, depending on the incident heat and particles fluxes and where these are deposited, consisting of concepts with different structural materials and service lifetimes. Preliminary analyses of such components are described in [8]. It is important that such components be integrated into the top of the machine in a way which enables them to be maintained without other plasma-facing components being removed.

\subsection{DN configuration}

If sections of the FW are simply too heavily loaded (in terms of heat loads and erosion) to be handled even by dedicated HHF 


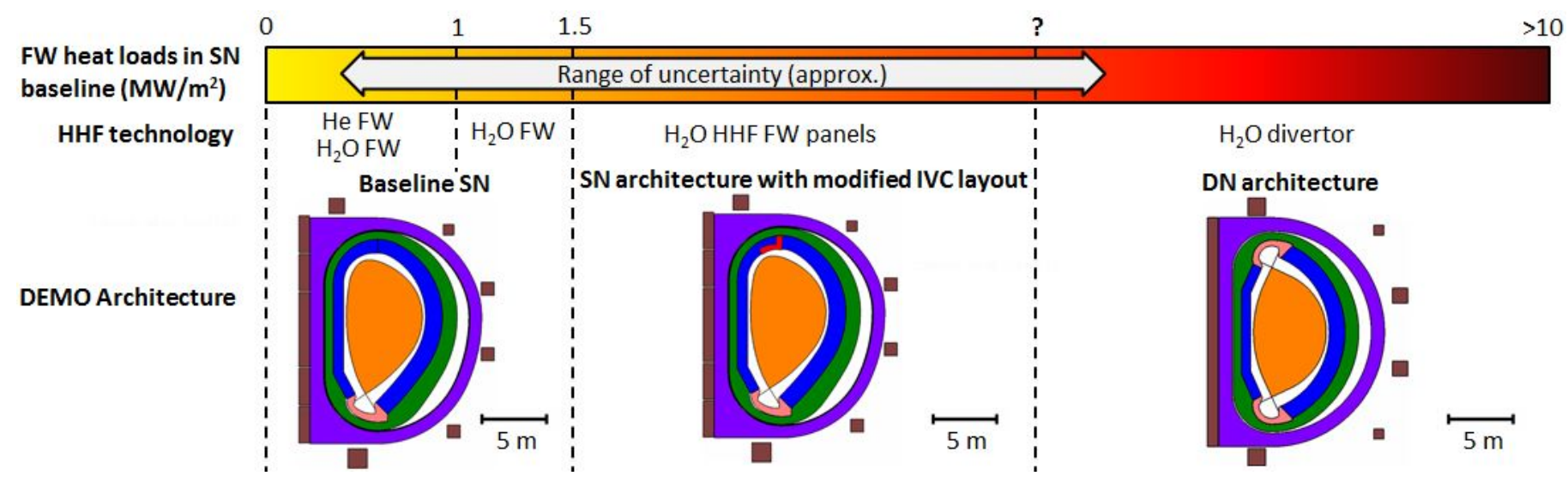

Figure 3: Uncertainty range of FW design heat loads at the top of the machine, entailing different HHF technology and DEMO architectural solutions. Note that the loads on the FW in the DN case are not expected to be high, rather that if loads on the FW exceed a certain limit, it could lead to a DN architecture, precisely to reduce the loads on the FW.

FW panels, a double-null configuration could be considered. The DN tokamak represents a significantly different PAM from the SN. The main advantages and disadvantages of such an architecture are yet to be fully quantified. Advantages possibly include an improved vertical stability, and a reduced heat flux to the divertor target plates of the lower divertor, since part of the SOL is intersected by the upper divertor, which will carry a significant part of the SOL heat loads. There are however downsides in terms of tritium breeding, and likely in overall plant efficiency and maintainability, too. Questions have also been raised regarding the erosion products of the upper divertor (dust) falling through the plasma, potentially causing disruptions.

\subsection{Comparison of architectures}

Following the procedure outlined in Figure 1, these alternative architectures have begun to be studied and compared against the baseline PAM (Figure 3).

The potential TBR was assessed for the DN in terms of the fraction of the plasma surface covered by the breeding blankets and the local breeding ratio, resulting in an estimated potential TBR of 1.12 for the DN, compared with 1.19 for the SN baseline [1]. For the SN case with dedicated HHF panels, the TBR will be lower than in the baseline (due to a thicker FW), but is likely to be higher than in the DN.

Data for further evaluations aspects will be compiled, including overall plant efficiency, maintainability, availability, plasma performance, reactor cost, etc. Results for these high level EAs would in some cases require many analyses to be carried out and, with limited resources, efforts must be concentrated on the systems which have the greatest effect on the performance of the different architectures.

Such an important decision on the direction of the DEMO architecture also requires the assessment of integration issues for each of the PAMs, such as those described in [3]. For example, the upper divertor's usage of space in the vertical port and the integration of pumps may prohibit the integration of $\mathrm{H} \& \mathrm{CD}$ systems in this port, driving up the required auxiliary power and usage of equatorial ports. Conversely, the vertical stability requirements in the DN may be relaxed, reducing the installed power required for the control of the plasma and opening the possibility to go to higher plasma elongations, potentially affecting the machine size.

\section{Summary and outlook}

Significant uncertainties in fusion science and engineering will persist throughout the DEMO conceptual design activities - in some cases for years to come. The direction of the DEMO architecture will need to be continually assessed during the conceptual design phase to address these uncertainties, and decisions will have to be made based on uncertain information. The importance of managing complexity and uncertainty has been highlighted, and a preliminary systems engineering framework for tackling uncertainties has been outlined; this will be developed further in future, with the aim of establishing a robust, repeatable, and traceable decision-making process for the DEMO architecture.

The issue of the uncertain FW loads at the top of the machine has been described, along with some preliminary considerations of alternatives, to illustrate architectural assessments in the face of uncertainty. Significant further work is needed in both the definition of the FW design heat loads (reduction of the uncertainties) and the evaluation of the alternative DEMO architectures, in particular the double-null.

\section{Acknowledgements}

This work has been carried out within the framework of the EUROfusion Consortium and has received funding from the Euratom research and training programme 2014-2018 under grant agreement No 633053. The views and opinions expressed herein do not necessarily reflect those of the European Commission.

\section{References}

[1] G. Federici et al., Overview of the design approach and prioritisation of R\&D activities towards an EU DEMO, Fusion Engineering and Design, this conference 
[2] M. Kovari et al., "PROCESS": A systems code for fusion power plants-Part 1: Physics, Fusion Engineering and Design 89 (2014) 30543069

[3] C. Bachmann et al., Issues and strategies for DEMO in-vessel component integration, Fusion Engineering and Design, this conference

[4] R. Wenninger et al., Advances in the physics basis for the European DEMO design, Nuclear Fusion 55 (2015).

[5] O. Crofts et al., Overview of Progress on the European DEMO Remote Maintenance Strategy, this conference

[6] R. Pitts et al., Physics basis and design of ITER plasma-facing components, Journal of Nuclear Materials (2011)

[7] R. Mitteau et al., The combined effects of magnetic asymmetry, assembly and manufacturing tolerances on the plasma loads to the ITER first wall, Journal of Nuclear Materials 463 (2015) 411-414

[8] T. Barrett, et al., Progress in the Engineering Design and Assessment of the European DEMO First Wall and Divertor Plasma Facing Components, Fusion Engineering and Design, this conference

[9] F. Maviglia et al., Development of DEMO wall heat load specification, IAEA Technical Meeting, Vienna, Austria 